\title{
Functions of several variables of finite variation and their differentiability
}

\author{
by DARIUSz IDCZAK (Łódź)
}

\begin{abstract}
Some differentiability properties of functions of several variables of finite variation are investigated.

1. Introduction. In the paper, nondecreasing functions of several variables and functions of several variables of finite variation on the closed unit interval are considered. A theorem on the Jordan decomposition and theorems on the measurability and differentiability of these functions are proved.

Nondecreasing functions of several variables, defined on the whole space, were studied in [1], [5]. A theorem on the nature of the set of discontinuity points of such functions was obtained in [1]. In [2] it was proved that a function nondecreasing with respect to each variable separately and defined on the whole space is differentiable a.e.

The definitions of a nondecreasing function of several variables and a function of several variables of finite variation, adopted in this paper, are analogous to the definition of an absolutely continuous function of several variables (cf. [6]). Our method of proof, similar to that in [6], is more elementary and shorter than that in [2].

To simplify notation, we consider the case of a function of two variables. In the last section the definitions of a nondecreasing function of $n$ variables and a function of $n$ variables of finite variation for $n>2$ are given. All theorems (with proofs) obtained for $n=2$ carry over to the case $n>2$.
\end{abstract}

2. Nondecreasing functions of two variables. Let $f$ be a real function of two real variables, defined on the interval

$$
[0,1] \times[0,1]=\left\{(x, y) \in \mathbb{R}^{2}: 0 \leq x, y \leq 1\right\}
$$

1991 Mathematics Subject Classification: 26B05, 26B30.

Key words and phrases: functions of several variables, nondecreasing functions, functions of finite variation, Fréchet differentiability. 
and let $F_{f}$ be the interval function defined by

$$
F_{f}(P)=f(\bar{x}, \bar{y})-f(\bar{x}, y)-f(x, \bar{y})+f(x, y)
$$

where $P=[x, \bar{x}] \times[y, \bar{y}] \subset[0,1] \times[0,1]$. The function $F_{f}$ is said to be associated with $f$.

We shall say that an interval function $F$ defined on intervals $P \subset[0,1] \times$ $[0,1]$ is additive if

$$
F(P \cup Q)=F(P)+F(Q)
$$

for all intervals $P, Q \subset[0,1] \times[0,1]$ such that $P \cup Q$ is an interval and Int $P \cap \operatorname{Int} Q=\emptyset$. Moreover, $F$ has a bounded variation if

$$
\sup \left\{\sum_{i=1}^{n}\left|F\left(P_{i}\right)\right|: \bigcup_{i=1}^{n} P_{i}=[0,1] \times[0,1], n \geq 1\right\}<\infty
$$

where $P_{i}, i=1, \ldots, n$, are intervals such that $\operatorname{Int} P_{i} \cap \operatorname{Int} P_{j}=\emptyset$ for $i \neq j$. Finally, $F$ has a derivative at $x \in[0,1] \times[0,1]$ if the limit

$$
\lim _{\substack{\delta(Q) \rightarrow 0 \\ x \in Q}} \frac{F(Q)}{|Q|}
$$

exists, where $Q \subset[0,1] \times[0,1]$ denotes a cube, $\delta(Q)$ the diameter of $Q$ and $|Q|$ the volume of $Q$.

In [3], it is proved that every nonnegative additive interval function and, consequently (by the Jordan decomposition), every interval function of bounded variation has a finite derivative a.e. on $[0,1] \times[0,1]$.

Definition 1. A function $f:[0,1] \times[0,1] \rightarrow \mathbb{R}$ will be called nondecreasing if $f(\cdot, 0), f(0, \cdot)$ are nondecreasing and the associated function $F_{f}$ is nonnegative.

From the above definition we directly obtain

Proposition 1. Let $f:[0,1] \times[0,1] \rightarrow \mathbb{R}$ be nondecreasing. Then $f\left(x_{1}, y_{1}\right) \leq f\left(x_{2}, y_{2}\right)$ for any $0 \leq x_{1} \leq x_{2} \leq 1$ and $0 \leq y_{1} \leq y_{2} \leq 1$.

Pro of. Let $0 \leq x_{1} \leq x_{2} \leq 1$ and $0 \leq y_{1} \leq y_{2} \leq 1$. We have

$$
\begin{aligned}
f\left(x_{1}, y_{1}\right) & =F_{f}\left(\left[0, x_{1}\right] \times\left[0, y_{1}\right]\right)+f\left(0, y_{1}\right)+f\left(x_{1}, 0\right)-f(0,0) \\
& \leq F_{f}\left(\left[0, x_{2}\right] \times\left[0, y_{2}\right]\right)+f\left(0, y_{2}\right)+f\left(x_{2}, 0\right)-f(0,0) \\
& =f\left(x_{2}, y_{2}\right) .
\end{aligned}
$$

This proposition immediately yields

COROllary 1. Every nondecreasing function $f:[0,1] \times[0,1] \rightarrow \mathbb{R}$ is bounded. 
The condition in the conclusion of Proposition 1 is equivalent to the following: for any $x, y \in[0,1]$, the functions $f(x, \cdot), f(\cdot, y)$ are nondecreasing. However, it does not imply that $F_{f}$ is nonnegative.

Now, we show that a nondecreasing function of two variables is measurable. This follows from Proposition 1 and

THEOREM 1. If $f:[0,1] \times[0,1] \rightarrow \mathbb{R}$ is nondecreasing with respect to each variable separately, then it is measurable.

Remark. This is a particular case of [4, Th. 2]. Here we give a direct proof.

Proof of Theorem 1 . Let $a \in \mathbb{R}$. We show that the set

$$
A=\{(x, y) \in[0,1] \times[0,1]: f(x, y)<a\}
$$

is measurable. Suppose that it is nonempty. Let

$$
B=\bigcup_{x \in[0,1]}\{x\} \times\left[0, \sup _{x}\right]
$$

where $\sup _{x}=\sup \{y \in[0,1]: f(x, y)<a\}$. Then

$$
x_{1}<x_{2} \Rightarrow \sup _{x_{1}} \geq \sup _{x_{2}} \text {. }
$$

Indeed, if $\sup _{x_{1}}<\sup _{x_{2}}$, then there would exist a constant $\alpha$ such that $\sup _{x_{1}}<\alpha<\sup _{x_{2}}$ and, consequently, $a \leq f\left(x_{1}, \alpha\right) \leq f\left(x_{2}, \alpha\right)<a$.

Thus the function $l:[0,1] \ni x \mapsto \sup _{x} \in[0,1]$ is nonincreasing and, consequently, measurable. From this (cf. [7]) it follows that the set

$$
C=\{(x, l(x)): x \in[0,1]\}
$$

has measure zero and the set $B$ is measurable.

Now, let $\left(x_{0}, y_{0}\right) \in A$, i.e. $f\left(x_{0}, y_{0}\right)<a$. Hence $y_{0} \in\left[0, \sup _{x_{0}}\right]$, that is, $\left(x_{0}, y_{0}\right) \in\left\{x_{0}\right\} \times\left[0, \sup _{x_{0}}\right] \subset B$. Thus $A \subset B$.

On the other hand, if

$$
\widetilde{B}=\bigcup_{x \in[0,1]}\{x\} \times\left[0, \sup _{x}[,\right.
$$

then $\widetilde{B} \subset A$. Indeed, let $\left(x_{0}, y_{0}\right) \in \widetilde{B}$. Then $y_{0} \in\left[0, \sup _{x_{0}}\left[\right.\right.$, that is, $f\left(x_{0}, y_{0}\right)$ $<a$, whence $\left(x_{0}, y_{0}\right) \in A$.

We have thus proved that $\widetilde{B} \subset A \subset B$, and the measurability of $A$ follows from the relations

$$
A=\widetilde{B} \cup(A \backslash \widetilde{B}), \quad \widetilde{B}=B \backslash C, \quad A \backslash \widetilde{B} \subset B \backslash \widetilde{B}=C .
$$

From the above theorem and Proposition 1 we have

COROllary 2. Every nondecreasing function $f:[0,1] \times[0,1] \rightarrow \mathbb{R}$ is measurable. 
3. Differentiability of nondecreasing functions. In this section we show that every nondecreasing function of two variables has the partial derivatives and the total differential a.e. We begin with the following

LEMMA 1. Let $f:[0,1] \rightarrow \mathbb{R}$ be nondecreasing, and $m \geq 3$ and $k \geq m$ be fixed positive integers. Then, for each $x \in] 1 / m, 1-1 / m[$,

$$
\inf _{0<|h|<1 / k} \frac{f(x+h)-f(x)}{h}=\inf _{0<|h|<1 / k, h \in \mathbb{Q}} \frac{f(x+h)-f(x)}{h}
$$

where $\mathbb{Q}$ denotes the set of rational numbers, and the same holds with inf replaced by sup.

Proof. It is sufficient to prove the inequality $\geq$. Let

$$
a:=\inf _{0<|h|<1 / k} \frac{f(x+h)-f(x)}{h} .
$$

There exists a sequence $\left(h_{n}\right)_{n \in \mathbb{N}}$ of numbers such that $0<\left|h_{n}\right|<1 / k$, $n \in \mathbb{N}$, and

$$
a=\lim _{n \rightarrow \infty} \frac{f\left(x+h_{n}\right)-f(x)}{h_{n}} .
$$

We shall show that, for any $n \in \mathbb{N}$, there exists a rational number $h_{n}^{q}$ such that $0<\left|h_{n}^{q}\right|<1 / k$ and

$$
\frac{f\left(x+h_{n}^{q}\right)-f(x)}{h_{n}^{q}} \leq \frac{f\left(x+h_{n}\right)-f(x)}{h_{n}}+\frac{1}{n} .
$$

First, assume that $h_{n}>0$. If $x+h_{n}$ is a left continuity point of $f$, then there exists a rational number $h_{n}^{q}$ such that $0<h_{n}^{q} \leq h_{n}$ and (1) holds. So, assume that

$$
\varepsilon:=f\left(x+h_{n}\right)-\lim _{h \rightarrow h_{n}^{-}} f(x+h)>0 .
$$

Let $h_{n}^{q} \in \mathbb{Q}$ be such that

$$
0 \leq h_{n} \frac{f\left(x+h_{n}\right)-f(x)}{f\left(x+h_{n}\right)-f(x)+\varepsilon}<h_{n}^{q} \leq h_{n} .
$$

Since $f$ is nondecreasing, by the monotonicity of $t \mapsto t /(t+\varepsilon)$ we have

$$
\frac{f\left(x+h_{n}^{q}\right)-f(x)}{f\left(x+h_{n}^{q}\right)-f(x)+\varepsilon} \leq \frac{f\left(x+h_{n}\right)-f(x)}{f\left(x+h_{n}\right)-f(x)+\varepsilon} .
$$

Now (2) gives

$$
h_{n} \frac{f\left(x+h_{n}^{q}\right)-f(x)}{f\left(x+h_{n}^{q}\right)-f(x)+\varepsilon} \leq h_{n} \frac{f\left(x+h_{n}\right)-f(x)}{f\left(x+h_{n}\right)-f(x)+\varepsilon}<h_{n}^{q} \leq h_{n} .
$$


We may assume that $0<f\left(x+h_{n}^{q}\right)-f(x)$ since otherwise (1) is obvious. Consequently,

$$
\begin{aligned}
\frac{f\left(x+h_{n}^{q}\right)-f(x)}{h_{n}^{q}} & \leq \frac{f\left(x+h_{n}^{q}\right)-f(x)}{h_{n} \frac{f\left(x+h_{n}^{q}\right)-f(x)}{f\left(x+h_{n}^{q}\right)-f(x)+\varepsilon}} \\
& =\frac{f\left(x+h_{n}^{q}\right)-f(x)+\varepsilon}{h_{n}} \leq \frac{f\left(x+h_{n}\right)-f(x)}{h_{n}}
\end{aligned}
$$

where the last inequality follows from the definition of $\varepsilon$. Thus (1) is satisfied.

Now, let $h_{n}<0$. If $x+h_{n}$ is a right continuity point of $f$, then there exists a rational number $h_{n}^{q}$ such that $h_{n} \leq h_{n}^{q}<0$ and (1) holds. So, assume that

$$
\delta:=\lim _{\bar{h} \rightarrow h_{n}^{+}} f(x+\bar{h})-f\left(x+h_{n}\right)>0 .
$$

Let $h_{n}^{q} \in \mathbb{Q}$ be such that

$$
h_{n} \leq h_{n}^{q}<h_{n} \frac{f\left(x+h_{n}\right)-f(x)}{f\left(x+h_{n}\right)-f(x)-\delta} \leq 0 .
$$

Since $f$ is nondecreasing, by the monotonicity of $t \mapsto t /(t-\delta)$ we have

$$
\frac{f\left(x+h_{n}^{q}\right)-f(x)}{f\left(x+h_{n}^{q}\right)-f(x)-\delta} \leq \frac{f\left(x+h_{n}\right)-f(x)}{f\left(x+h_{n}\right)-f(x)-\delta} .
$$

Now (3) yields

$$
h_{n} \leq h_{n}^{q}<h_{n} \frac{f\left(x+h_{n}\right)-f(x)}{f\left(x+h_{n}\right)-f(x)-\delta} \leq h_{n} \frac{f\left(x+h_{n}^{q}\right)-f(x)}{f\left(x+h_{n}^{q}\right)-f(x)-\delta} .
$$

We may assume that $f\left(x+h_{n}^{q}\right)-f(x)<0$ since otherwise (1) is obvious. Consequently,

$$
\begin{aligned}
\frac{f\left(x+h_{n}^{q}\right)-f(x)}{h_{n}^{q}} & =\frac{-f\left(x+h_{n}^{q}\right)+f(x)}{\left|h_{n}^{q}\right|} \leq \frac{-f\left(x+h_{n}^{q}\right)+f(x)}{\left|h_{n}\right| \frac{f\left(x+h_{n}^{q}\right)-f(x)}{f\left(x+h_{n}^{q}\right)-f(x)-\delta}} \\
& =\frac{f\left(x+h_{n}^{q}\right)-f(x)-\delta}{-\left|h_{n}\right|}=\frac{-f\left(x+h_{n}^{q}\right)+f(x)+\delta}{\left|h_{n}\right|} \\
& \leq \frac{-f\left(x+h_{n}\right)+f(x)}{\left|h_{n}\right|}=\frac{f\left(x+h_{n}\right)-f(x)}{h_{n}}
\end{aligned}
$$

where the last inequality follows from the definition of $\delta$. Thus condition (1) is satisfied.

Of course, by the definition of $a$, for any $n \in \mathbb{N}$,

$$
a \leq \frac{f\left(x+h_{n}^{q}\right)-f(x)}{h_{n}^{q}} .
$$


So, (1) gives

$$
\lim _{n \rightarrow \infty} \frac{f\left(x+h_{n}^{q}\right)-f(x)}{h_{n}^{q}}=a .
$$

Thus

$$
\inf _{0<|h|<1 / k, h \in \mathbb{Q}} \frac{f(x+h)-f(x)}{h} \leq a .
$$

The proof of the "sup" version is analogous.

Now, we have

Proposition 2. Let $f:[0,1] \times[0,1] \rightarrow \mathbb{R}$ be measurable. If it is nondecreasing with respect to the first variable, then $\frac{\partial f}{\partial x}(x, y)$ exists a.e. on $[0,1] \times[0,1]$

Proof. We show that $\frac{\partial f}{\partial x}(x, y)$ exists a.e. on $] 0,1[\times[0,1]$. For any $m \geq 3$, and $k=m, m+1, \ldots$, we define

$$
\begin{aligned}
& \left.\phi_{k}^{m}:\right] 1 / m, 1-1 / m\left[\times[0,1] \ni(x, y) \mapsto \inf _{0<|h|<1 / k} \frac{f(x+h, y)-f(x, y)}{h} \in \overline{\mathbb{R}},\right. \\
& \left.\psi_{k}^{m}:\right] 1 / m, 1-1 / m\left[\times[0,1] \ni(x, y) \mapsto \sup _{0<|h|<1 / k} \frac{f(x+h, y)-f(x, y)}{h} \in \overline{\mathbb{R}} .\right.
\end{aligned}
$$

It is easy to see that the set of all points $(x, y) \in] 1 / m, 1-1 / m[\times[0,1]$ at which $\frac{\partial f}{\partial x}(x, y)$ exists is equal to

$$
\begin{aligned}
E^{m}=\{(x, y) \in] 1 / m, 1 & -1 / m[\times[0,1]: \\
& \left.-\infty<\lim _{k \rightarrow \infty} \phi_{k}^{m}(x, y)=\lim _{k \rightarrow \infty} \psi_{k}^{m}(x, y)<\infty\right\} .
\end{aligned}
$$

From Lemma 1 it follows that the infimum and supremum in the definitions of $\phi_{k}^{m}$ and $\psi_{k}^{m}$ can be restricted to rational $h$. Since $f$ is measurable and $\mathbb{Q}$ is countable, this means that $E^{m}$ is measurable.

Since the set $\widetilde{E}$ of all points of $] 0,1\left[\times[0,1]\right.$ at which $\frac{\partial f}{\partial x}(x, y)$ exists is equal to $\bigcup_{m>3} E^{m}$, it follows that $\widetilde{E}$ is measurable.

Since $f(\cdot, y)$ is nondecreasing for every $y$ and, consequently, is differentiable a.e. on $[0,1]$, all $y$-sections of $\widetilde{E}$ have full measure. This shows that $\widetilde{E}$ has full measure.

From Proposition 2 and Theorem 1 we obtain

THEOREM 2. If $f:[0,1] \times[0,1] \rightarrow \mathbb{R}$ is nondecreasing with respect to each variable separately, then $\frac{\partial f}{\partial x}(x, y)$ and $\frac{\partial f}{\partial y}(x, y)$ exist a.e. on $[0,1] \times[0,1]$.

This theorem and Proposition 1 yield

Corollary 3. If $f:[0,1] \times[0,1] \rightarrow \mathbb{R}$ is nondecreasing, then $\frac{\partial f}{\partial x}(x, y)$ and $\frac{\partial f}{\partial y}(x, y)$ exist a.e. on $[0,1] \times[0,1]$.

Now, we shall prove 
THEOREM 3. If $f:[0,1] \times[0,1] \rightarrow \mathbb{R}$ is nondecreasing, then it has the total differential a.e. on $[0,1] \times[0,1]$.

Proof. Denote by $E$ the set (of full measure) of all points at which $\frac{\partial f}{\partial x}(x, y), \frac{\partial f}{\partial y}(x, y)$ and $D F_{f}(x, y)$ all exist. Let $(x, y) \in E$ and put

$$
A^{1}=\frac{\partial f}{\partial x}(x, y), \quad A^{2}=\frac{\partial f}{\partial y}(x, y)
$$

We show that

$$
\frac{f\left(x+h^{1}, y+h^{2}\right)-f(x, y)-A^{1} h^{1}-A^{2} h^{2}}{\left|\left(h^{1}, h^{2}\right)\right|} \rightarrow 0 \quad \text { as }\left(h^{1}, h^{2}\right) \rightarrow 0 .
$$

Without loss of generality we may assume that $h^{1} \neq 0$ and $h^{2} \neq 0$ (if $h^{1}=0$ or $h^{2}=0$, then the convergence follows from the existence of the partial derivatives at $(x, y))$. Thus

$$
\begin{aligned}
&\left.\frac{f\left(x+h^{1}, y+\right.}{}+h^{2}\right)-f(x, y)-A^{1} h^{1}-A^{2} h^{2} \\
&= \frac{f\left(x+h^{1}, y+h^{2}\right)-f(x, y)-f\left(x+h^{1}, y\right)+f(x, y)}{\left|\left(h^{1}, h^{2}\right)\right|} \\
&+\frac{\alpha\left(x, h^{1}\right) h^{1}-f\left(x, y+h^{2}\right)+f(x, y)+\beta\left(y, h^{2}\right) h^{2}}{\left|\left(h^{1}, h^{2}\right)\right|} \\
&= \frac{f\left(x+h^{1}, y+h^{2}\right)-f\left(x+h^{1}, y\right)-f\left(x, y+h^{2}\right)+f(x, y)}{\sqrt{\left(h^{1}\right)^{2}+\left(h^{2}\right)^{2}}} \\
&+\frac{\alpha\left(x, h^{1}\right) h^{1}}{\sqrt{\left(h^{1}\right)^{2}+\left(h^{2}\right)^{2}}}+\frac{\beta\left(y, h^{2}\right) h^{2}}{\sqrt{\left(h^{1}\right)^{2}+\left(h^{2}\right)^{2}}} \\
&= \frac{F_{f}\left(\left[x, x+h^{1}\right] \times\left[y, y+h^{2}\right]\right)}{\sqrt{\left(h^{1}\right)^{2}+\left(h^{2}\right)^{2}}}+\frac{\alpha\left(x, h^{1}\right) h^{1}}{\sqrt{\left(h^{1}\right)^{2}+\left(h^{2}\right)^{2}}} \\
&+\frac{\beta\left(y, h^{2}\right) h^{2}}{\sqrt{\left(h^{1}\right)^{2}+\left(h^{2}\right)^{2}}}
\end{aligned}
$$

where $\alpha\left(x, h^{1}\right) \rightarrow 0$ as $h^{1} \rightarrow 0$, and $\beta\left(y, h^{2}\right) \rightarrow 0$ as $h^{2} \rightarrow 0$.

Since

$$
\begin{gathered}
\frac{\left|\alpha\left(x, h^{1}\right) h^{1}\right|}{\sqrt{\left(h^{1}\right)^{2}+\left(h^{2}\right)^{2}}} \leq\left|\alpha\left(x, h^{1}\right)\right| \rightarrow 0, \\
\frac{\left|\beta\left(y, h^{2}\right) h^{2}\right|}{\sqrt{\left(h^{1}\right)^{2}+\left(h^{2}\right)^{2}}} \rightarrow 0,
\end{gathered}
$$


and

$$
\frac{\left|h^{1}\right|\left|h^{2}\right|}{\sqrt{\left(h^{1}\right)^{2}+\left(h^{2}\right)^{2}}} \leq \frac{\sqrt{\left(h^{1}\right)^{2}+\left(h^{2}\right)^{2}} \sqrt{\left(h^{1}\right)^{2}+\left(h^{2}\right)^{2}}}{\sqrt{\left(h^{1}\right)^{2}+\left(h^{2}\right)^{2}}} \rightarrow 0,
$$

as $\left(h^{1}, h^{2}\right) \rightarrow 0$, it suffices to show that

$$
\frac{F_{f}\left(\left[x, x+h^{1}\right] \times\left[y, y+h^{2}\right]\right)}{\sqrt{\left(h^{1}\right)^{2}+\left(h^{2}\right)^{2}}} \rightarrow 0 \quad \text { as }\left(h^{1}, h^{2}\right) \rightarrow 0 .
$$

Indeed, let $K \subset[0,1] \times[0,1]$ be any cube containing $\left[x, x+h^{1}\right] \times\left[y, y+h^{2}\right]$, with edge length $\max \left(\left|h^{1}\right|,\left|h^{2}\right|\right)$. Then

$$
\begin{aligned}
0 & \leq \frac{F_{f}\left(\left[x, x+h^{1}\right] \times\left[y, y+h^{2}\right]\right)}{\sqrt{\left(h^{1}\right)^{2}+\left(h^{2}\right)^{2}}} \leq \frac{F_{f}(K)}{\sqrt{\left(h^{1}\right)^{2}+\left(h^{2}\right)^{2}}} \\
& =\frac{F_{f}(K)}{\left(\max \left(\left|h^{1}\right|,\left|h^{2}\right|\right)\right)^{2}} \cdot \frac{\left(\max \left(\left|h^{1}\right|,\left|h^{2}\right|\right)\right)^{2}}{\sqrt{\left(h^{1}\right)^{2}+\left(h^{2}\right)^{2}}} \\
& =\frac{F_{f}(K)}{|K|} \cdot \frac{\left(\max \left(\left|h^{1}\right|,\left|h^{2}\right|\right)\right)^{2}}{\sqrt{\left(h^{1}\right)^{2}+\left(h^{2}\right)^{2}}} \\
& \leq \frac{F_{f}(K)}{|K|} \cdot \frac{\left(\sqrt{\left(h^{1}\right)^{2}+\left(h^{2}\right)^{2}}\right)^{2}}{\sqrt{\left(h^{1}\right)^{2}+\left(h^{2}\right)^{2}}} \rightarrow D F_{f}(x, y) \cdot 0
\end{aligned}
$$

as $\left(h^{1}, h^{2}\right) \rightarrow 0$. Hence $f$ is differentiable at $(x, y)$ with total differential

$$
\mathbb{R}^{2} \ni\left(h^{1}, h^{2}\right) \mapsto \frac{\partial f}{\partial x}(x, y) h^{1}+\frac{\partial f}{\partial y}(x, y) h^{2} .
$$

4. Functions of two variables of finite variation. In this section we give a definition of a function of two variables of finite variation. We show that, as in the case of one variable, such a function can be written as the difference of two nondecreasing functions. This yields the a.e. existence of the total differential of such functions.

Definition 2. A function $f:[0,1] \times[0,1] \rightarrow \mathbb{R}$ is said to be of finite variation if $f(\cdot, 0), f(0, \cdot)$ are of finite variation and the associated function $F_{f}$ has a finite variation.

The proof of the next theorem is based on Jordan's decomposition of a function of one variable of finite variation and of an interval function of finite variation.

TheOREM 4. A function $f:[0,1] \times[0,1] \rightarrow \mathbb{R}$ has a finite variation if and only if there exist nondecreasing functions $g, h:[0,1] \times[0,1] \rightarrow \mathbb{R}$ such that $f=g-h$.

Proof. Necessity. Let $f$ have a finite variation. Then

$$
f(x, y)=f(x, 0)+f(0, y)-f(0,0)+F_{f}([0, x] \times[0, y])
$$


for $(x, y) \in[0,1] \times[0,1]$. Let $g^{1}, h^{1}, g^{2}, h^{2}$ be nondecreasing functions of one variable such that

$$
\begin{array}{ll}
f(x, 0)=g^{1}(x)-h^{1}(x), & x \in[0,1], \\
f(0, y)=g^{2}(y)-h^{2}(y), & y \in[0,1],
\end{array}
$$

and $G, H$ additive nonnegative interval functions such that

$$
F_{f}(P)=G(P)-H(P), \quad P \subset[0,1] \times[0,1] .
$$

Put

$$
\begin{aligned}
& g(x, y)=g^{1}(x)+g^{2}(y)-\frac{1}{2} f(0,0)+G([0, x] \times[0, y]), \\
& h(x, y)=h^{1}(x)+h^{2}(y)+\frac{1}{2} f(0,0)+H([0, x] \times[0, y]),
\end{aligned}
$$

for $(x, y) \in[0,1] \times[0,1]$. Thus $f(x, y)=g(x, y)-h(x, y)$ for $(x, y) \in[0,1] \times$ $[0,1]$.

The functions $g, h$ are nondecreasing. Indeed, since

$$
\begin{aligned}
& g(0, y)=g^{1}(0)+g^{2}(y)-\frac{1}{2} f(0,0), \\
& g(x, 0)=g^{1}(x)+g^{2}(0)-\frac{1}{2} f(0,0)
\end{aligned}
$$

for $(x, y) \in[0,1] \times[0,1]$ and, by the definition of $F_{g}$,

$$
F_{g}(P)=G(P)
$$

for any interval $P \subset[0,1] \times[0,1]$, it follows that $g$ is nondecreasing. The proof for $h$ is similar.

Sufficiency. In this case,

$$
\begin{aligned}
f(0, y) & =g(0, y)-h(0, y), & & y \in[0,1], \\
f(x, 0) & =g(x, 0)-h(x, 0), & & x \in[0,1], \\
F_{f}(P) & =F_{g}(P)-F_{h}(P), & & P \subset[0,1] \times[0,1],
\end{aligned}
$$

and the assertion is obvious.

The above theorem and the results of Sections 1-3 yield the following results:

Every function $f:[0,1] \times[0,1] \rightarrow \mathbb{R}$ of finite variation is bounded.

If $f:[0,1] \times[0,1] \rightarrow \mathbb{R}$ has a finite variation, then, for any $x, y \in[0,1]$, the functions $f(x, \cdot), f(\cdot, y)$ are of finite variation.

Every function $f:[0,1] \times[0,1] \rightarrow \mathbb{R}$ of finite variation is measurable.

If $f:[0,1] \times[0,1] \rightarrow \mathbb{R}$ is of finite variation, then $\frac{\partial f}{\partial x}(x, y)$ and $\frac{\partial f}{\partial y}(x, y)$ exist a.e. on $[0,1] \times[0,1]$.

THEOREM 5. If $f:[0,1] \times[0,1] \rightarrow \mathbb{R}$ is of finite variation, then it has the total differential a.e. on $[0,1] \times[0,1]$.

This is an extension of the theorem on the differentiability a.e. of an absolutely continuous function of two variables (cf. [6]). 
5. The case $n>2$. Our definitions of a nondecreasing function and of a function of finite variation can be extended by induction to the case of $n$ variables, $n>2$. Without going into details, it can be said that a real function $f$ is nondecreasing (resp. of finite variation) on

$$
K^{n}=\left\{x=\left(x^{1}, \ldots, x^{n}\right) \in \mathbb{R}^{n}: 0 \leq x^{i} \leq 1, i=1, \ldots, n\right\}
$$

if the associated function $F_{f}(P), P \subset K^{n}$ (cf.[3]), is nonnegative (resp. of finite variation) and each of the functions $f\left(0, x^{2}, \ldots, x^{n}\right), f\left(x^{1}, 0, x^{3}, \ldots\right.$ $\left.\ldots, x^{n}\right), \ldots, f\left(x^{1}, \ldots, x^{n-1}, 0\right)$ is a nondecreasing function of $n-1$ variables (resp. a function of $n-1$ variables of finite variation).

As mentioned in the introduction, the above definitions allow us to easily carry over all the theorems (with proofs) obtained in the paper to the case $n>2$.

\section{References}

[1] P. Antosik, The investigation of the continuity of functions of several variables, Comment. Math. 10 (1966), 101-104 (in Russian).

[2] Y. Chabrillac and J. P. Crouzeix, Continuity and differentiability properties of monotone real functions of several real variables, Math. Programming Study 30 (1987), 1-16.

[3] S. Łojasiewicz, An Introduction to the Theory of Real Functions, Wiley, Chichester, 1988.

[4] E. Marczewski et C. Ryll-Nardzewski, Sur la mesurabilité des fonctions de plusieurs variables, Ann. Soc. Polon. Math. 25 (1952), 145-149.

[5] S. Mazurkiewicz, Foundations of Probability, PWN, Warszawa, 1956 (in Polish).

[6] S. Walczak, On the differentiability of absolutely continuous functions of several variables. Remarks on the Rademacher theorem, Bull. Polish Acad. Sci. Math. 36 (1988), 513-520.

[7] R. L. Wheeden and A. Zygmund, Measure and Integral: an Introduction to Real Analysis, Marcel Dekker, 1977.

INSTITUTE OF MATHEMATICS

UNIVERSITY OF ŁÓDŹ

BANACHA 22

90-238 LÓDŹ, POLAND 